\title{
Optimum Arrival Routes for Flight Efficiency
}

\author{
Ozlem Sahin Meric \\ Faculty of Aeronautics and Astronautics, Eskisehir, Turkey \\ Email: osahin5@anadolu.edu.tr
}

Received January 2015

\begin{abstract}
With the development of aircraft equipment, conventional navigation is the shift from performance based navigation (PBN). As is known, conventional navigation is based on ground-based navigation aids; however, $\mathrm{PBN}$ is based on aircraft avionics and performance. In this paper, a new method called Point Merge System (PMS) considered as one of PBN procedures will be introduced. PMS has many benefits related to fuel savings and emission reductions by implementing Continuous Descent Approaches (CDAs). A new PMS standard arrival route (STAR) model will be designed in radar simulation and it will be suggested.
\end{abstract}

\section{Keywords}

Performance Based Navigation, Area Navigation, Required Navigation Performance, Point Merge System, Efficiency

\section{Introduction}

Open loop radar vectors (tactical vectoring) included heading instructions are a common used method for merging and handling arrival traffic flows. This method is efficient and flexible but in the high traffic density areas, the controller should give rapid decisions for each aircraft in a critical time window. Therefore, controller's workload and frequency occupancy time are increased. Also it is difficult to provide optimum flight profiles and to control traffic dispersion [1]. Moreover, standard arrival routes (STARs) based on conventional procedures could be used for handling arrival traffic. Aircraft should follow a route which is described by ground navigational aid. Due to stay dependent on ground, it limits providing more direct routing.

With the development of satellite based technology and aircraft equipment, conventional flight procedures are shift from performance based navigation (PBN). As is known, conventional procedures are based on groundbased navigation aids; however, PBN is based on aircraft avionics and performance. PBN consists of Area Navigation (RNAV) and Required Navigation Performance (RNP) specifications. RNAV is defined as "a method of navigation which permits aircraft operation on any desired flight path within the coverage of station-referenced navigation aids or within the limits of the capability of self contained aids, or a combination of these" (ICAO Annex 11, 2001) [2]. RNP is similar but it enables alerting and monitoring system on board namely it is the key difference between RNAV and RNP [3] [4]. More flexible arrival and approach procedures which allow the efficient use of airspace could be designed by using RNAV route structure. In order to maximize capacity, sometimes, RNAV routes could be combined with conventional procedures. In that case, controller can begin to use heading instructions. Today, instead of conventional procedures, RNAV procedures, which provide effective 
and efficient use of TMA, are preferred to be used for handling and sequencing arrival traffic operations.

A new method called point merge system (PMS) is based on RNAV route structure. PMS intends merging arrival traffic flows on a point. And also it provides path stretching or shortening by using available predefined legs.

PMS doesn't need any heading instructions. Traffic follows a standard arrival route which enables continuous descent approaches (CDAs) [5]. CDA is defined as a method that the aircraft intercepts glide path from optimal vertical profile down to touchdown with engines operating at low thrust power [6]. The most common benefits of CDA procedures are reducing aircraft noise, fuel burn and emissions [7]. Reduction in frequency occupancy time and workload, also by enabling CDA, reduction in fuel consumption and consequently reduction in emissions are the expected benefits of PMS procedure [5]. In the following part below, PMS and CDA will be reviewed in terms of fuel savings and emissions.

Clarke et al. (2004) made a study related to CDA procedures. It was tested by simulations that in Louisville International Airport indicated 400 to $500 \mathrm{lb}(181-227 \mathrm{~kg})$ of lower fuel consumption during approach [8].

Ivanescu et al., assessed the performance of PMS and compared with vectoring in fast time simulator. In that study, $20 \%$ reduction in workload, approximately $30 \%$ reduction in number of controller instructions and 170 $\mathrm{kg} \pm 14 \mathrm{~kg}$ reduction in fuel burn were obtained [9].

Another study reporting fuel savings due to CDA was made by Turgut et al. (2010). Conventional and CDA procedures were compared by using real flight data for B757 and more than $40 \mathrm{~kg}$ fuel and 2 minutes time savings were reported for CDA procedures [6]. Robinson (2010) studied on different CDA scenarios and the results of simulation indicated $50 \mathrm{~kg}-150 \mathrm{~kg}$ fuel savings at medium or low traffic density [10].

PMS has been applied in Oslo in 2011. The results for Oslo present that PMS has more advantages over vectoring such as providing more efficient and predictable way, reducing workload, improving safety and minimizing environmental impacts by allowing CDA. The results indicate $300 \mathrm{~kg}$ reduction per flight in $\mathrm{CO}_{2}$ emissions [11].

At Ahmadabad Airport in India, CDA procedures have been implemented. Fuel savings about 1164 tonnes and 3678 tonnes $\mathrm{CO}_{2}$ emission reduction annually were reported. Moreover, another study related to CDA was performed in Praque. It is addressed that 65 - $96 \mathrm{~kg}$ fuel savings and $200-300 \mathrm{~kg} \mathrm{CO}$ reduction per flight could be estimated and it corresponds to 1400 tons fuel savings and 4600 tons $\mathrm{CO}_{2}$ reductions annually [12].

The previous studies given above stress a significant reduction in fuel consumption and in $\mathrm{CO}_{2}$ emissions by implementing PMS CDA procedures in TMA. In this study, PMS STAR model proposal is recommended for both Istanbul Ataturk International Airport (LTBA) and Sabiha Gokcen Airport (LTFJ).

\section{Point Merge System (PMS)}

PMS is a sequencing method which is based on RNAV route and it is used in transition or initial approach procedure or a portion thereof. PMS could be defined as a RNAV STAR. In other words PMS could be introduced as a method handling arrival traffic flows without heading instructions [1].

A single point and predefined legs are the elements of PMS. This method aims at integrating arrival traffic flows on a single point called merge point. Before merge point, by using predefined legs-called sequencing legs-collecting arrival traffic from different directions could be achieved (Figure 1). These legs could be placed in the same distance from the merge point (eqidistant) and traffic remain on the same distance to merge point during flight on these legs (isodistant) [5].

While operating PMS procedure, aircraft remain on sequencing legs until controllers give a "direct to" instruction to the merge point. After leaving the leg, aircraft might begin descending and controller use speed control instructions in order to provide in trail separation. By using PMS STAR procedures, descent profiles could be optimized by implementing CDA.

\section{A PMS STAR Model Proposal}

International Istanbul Ataturk Airport (LTBA) and Sabiha Gokcen Airport (LTFJ) are in Istanbul terminal control area (TMA) and are close each other. RNAV STAR has been implemented for handling and managing arrival traffic flows. For landing, 05/23 and 06/24 runway configuration is usually used for LTBA and LTFJ, respectively.

In this study, different entry points to Istanbul TMA located separately for collecting arrival traffic and called 
ENT $\times$ (i.e. ENT 1, ENT 2, ..). Merge points and sequencing legs are located in an appropriate point which allow intercepting ILS course to related runways by implementing CDA procedures. Merge points are called Tx. T2 and T3 are used for LTBA and LTFJ, respectively. However T1 is used for both LTBA and LTFJ. T4 is the common point which is to be followed after PMS procedures. T1 and T2 is located unsymmetrically configuration so as to prevent nose to nose situation on T4. PMS procedures which include T1 and T2 is an example of parallel combination of a PMS. Another is the serial combination and in the recommended PMS STAR model, it could be seen at the north region of TMA. T merge point could be introduced as a feeder waypoint which helps collecting high traffic flows to PMS procedure included T1. In order to meet high traffic demand, the number of sequencing legs could be increased or multiple combination of PMS could be used. As mentioned above, in this study for LTBA serial and parallel combination of PMS is preferred in design instead of increasing number of sequencing legs.

Using PMS STAR, traffic are delayed on sequencing legs instead of holding on a point or navigational aids. After reaching at the end of the procedure, traffic could turn to merge point automatically and also starts descending. Initial waypoints for joining the PMS procedure are presented orange unfilled points, however, the last waypoints of procedures are illustrated in filled orange. Moreover sequencing legs and the links of ATS arrival routes are showed in blue (Figure 2).

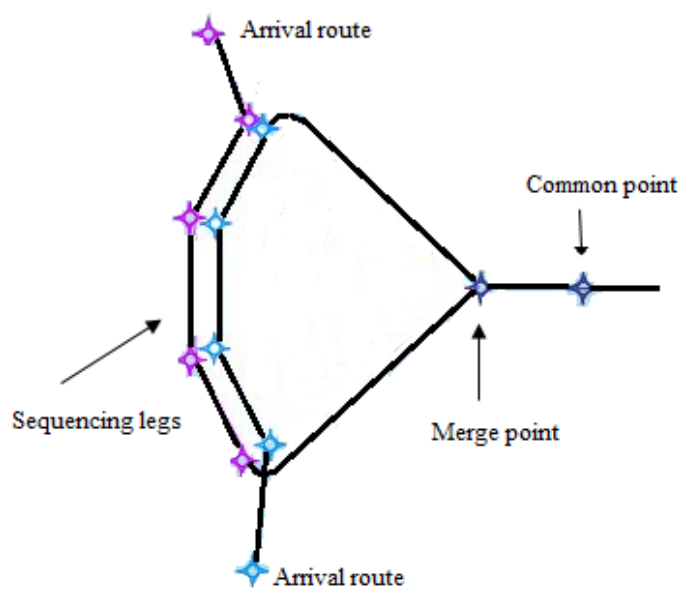

Figure 1. PMS route structure [5].

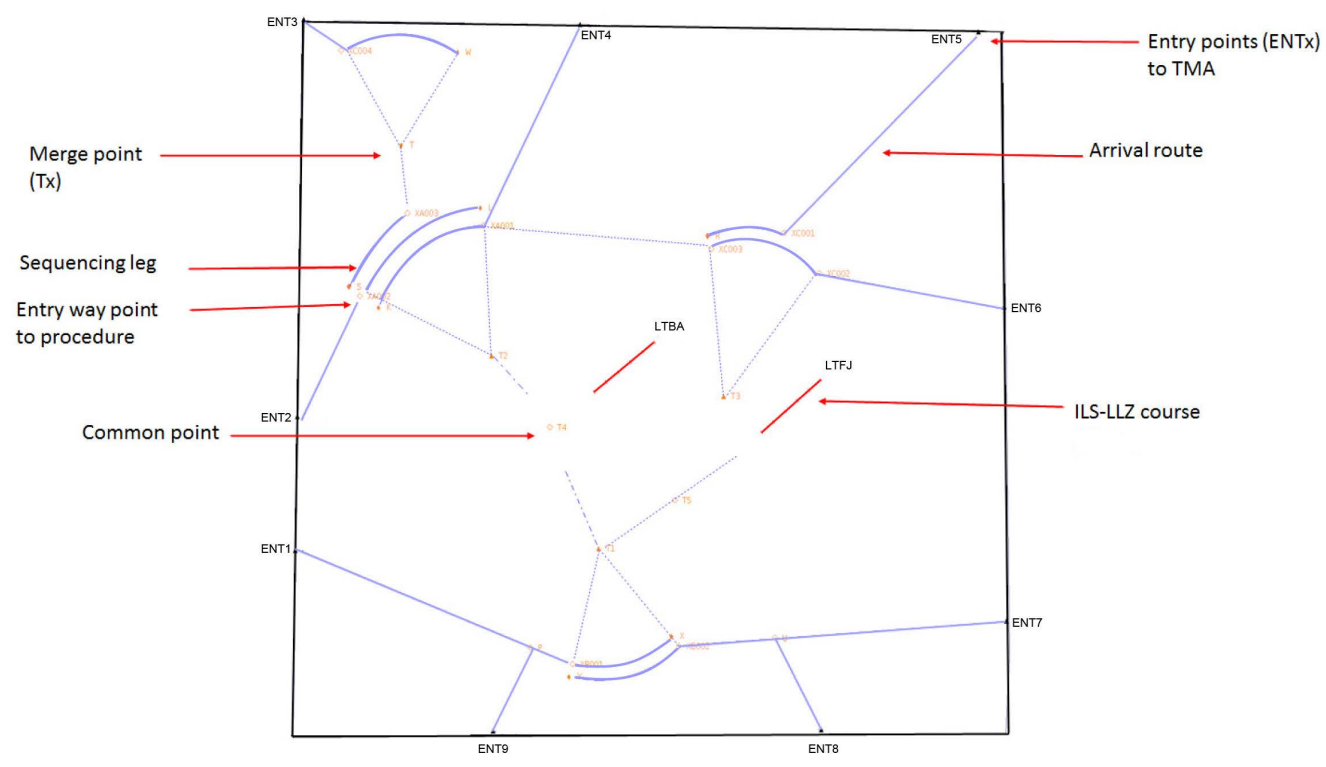

Figure 2. A proposed PMS STAR model for LTBA and LTFJ. 


\section{Conclusion}

PMS, which is based on RNAV, is used as a method for sequencing and integrating arrival traffic and aims to increase safety, flight efficiency and predictability. PMS procedures allow aircraft to follow more direct routings which cause a reduction in flight time and distance. Also, PMS allows CDA procedures which help to optimize the descent profiles. Implementing CDA, traffic proposes to descent with low drag and low thrust, and also keep higher altitude at longer time as compared with conventional procedures. As a result, it minimizes the environmental impacts such as a reduction in fuel consumption and consequently $\mathrm{CO}_{2}$ emissions. In this study, a PMS STAR model included different design combinations of PMS is suggested for LTBA and LTFJ airports. By using the proposed new model in the terminal airspace, flight efficiency and predictability might be increased and expected benefits of PMS could be appeared. In the future study, by using the real flight data, proposed PMS STAR model will be compared with baseline procedures and results will be assessed in terms of flight time, flight distance, fuel consumption and emissions.

\section{References}

[1] Boursier, L., Favennec, B., Hoffman, E., Trzmiel, A., Vergne, F. and Zeghal, K. (2007) Merging Arrival Flows without Heading Instructions. 7th USA/Europe Air Traffic Management R\&D Seminar, Barcelona.

[2] International Civil Aviation Organization (ICAO) (2001) Annex 11. Air Traffic Services. 13th Edition.

[3] International Civil Aviation Organization (ICAO) (2008) Doc 9613. Performance-Based Navigation (PBN) Manual. 3rd Edition.

[4] International Civil Aviation Organization (ICAO) (2009) Doc 9905. Required Navigation Performance Authorization Required (RNP AR) Procedure Design Manual.

[5] EUROCONTROL (2010) Point Merge Integration of Arrival Flows Enabling Extensive RNAV Application and CDA-Operational Services and Environment Definition. Eurocontrol Experimental Center, Version 2.0.

[6] Turgut, E.T., Usanmaz, Ö., Canarslanlar, A.O. and Sahin, Ö. (2010) Energy and Emission Assessment of Continuous Descent Approach. Aircraft Engineering and Aerospace Technology: An International Journal, 82, 32-38. http://dx.doi.org/10.1108/00022661011028092

[7] Turgut, E.T. (2011) Estimating Aircraft Fuel Flow for a Three-Degree Flight-Path-Angle Descent. Journal of Aircraft, 48, 1099-1106. http://dx.doi.org/10.2514/1.C031260

[8] Clarke, J.P.B., Ho, N.T., Ren, L., Brown, J.A., Elmer, K.R., Tong, K. and Wat, J.K. (2004) Continuous Descent Approach: Design and Fight Test for Louisville International Airport. Journal of Aircraft, 41, 1054-1066. http://dx.doi.org/10.2514/1.5572

[9] Ivanescu, D., Shaw, C., Tamvaclis, C. and Kettunen, T. (2009) Models of Air Traffic Merging Techniques: Evaluating Performance of Point Merge. 9th AIAA Aviation Technology, Integration, and Operations Conference (ATIO).

[10] Robinson, J.E. (2010) Benefits of Continuous Descent Operations in High-Density Terminal Airspace under Scheduling Constraints. 10th AIAA Aviation Technology, Integration, and Operations Conference (ATIO).

[11] http://www.isa-software.com/point-merge-system-deployed-in-oslo/

[12] International Civil Aviation Organization (ICAO) (2014) Capacity and Efficiency Air Navigation Report. http://www.icao.int/airnavigation/Documents/ICAO_AN\%20Report_EN_final_30042014.pdf 